\title{
Unbounded generalization of logarithmic representation of infinitesimal generators
}

\author{
Yoritaka Iwata ${ }^{1}$ \\ ${ }^{1}$ Kansai University
}

January 20, 2021

\begin{abstract}
The logarithmic representation of infinitesimal generators is generalized to the cases when the evolution operator is unbounded. The generalized result is applicable to the representation of infinitesimal generators of unbounded evolution operators, where unboundedness of evolution operator is an essential ingredient of nonlinear analysis. In conclusion a general framework for the identification between the infinitesimal generators with evolution operators is established. A mathematical framework for such an identification is indispensable to the rigorous treatment of nonlinear transforms: e.g., transforms appearing in the theory of integrable systems.
\end{abstract}

\section{Hosted file}

iwata-manuscript_27.pdf available at https://authorea.com/users/390691/articles/504930unbounded-generalization-of-logarithmic-representation-of-infinitesimal-generators 